Supplement Issue 1: International Tehran Breast Cancer Congress (TBCC9)

\title{
Dissection of Gene Expression Profile in Breast Cancer Cells Treated with Cisplatin in two Glycolysis and Impedance Steps
}

\author{
Rasoul Godini ${ }^{1,{ }^{*}}$, Hossein Fallahi ${ }^{1}$ \\ ${ }^{1}$ Department of Biology, Faculty of science, Razi University, Kermanshah, \\ Kermanshah, Iran
}

${ }^{*}$ Corresponding Author: Rasoul Godini, Department of Biology, Faculty of Science,

DOI: $10.21859 /$ mci-supp-39 Razi University, Kermanshah, Kermanshah, Iran.E-mail: rasoul_godini@yahoo.com

\author{
Keywords: \\ Breast Cancer \\ Cell line \\ Cisplatin \\ Gene Expression
}

\begin{abstract}
Introduction Breast cancer (BC) is one of the most common cancer types in women, causing death in many cases. Cisplatin is one of the drugs for treating the disease that induce cell death in a few hours after treatment. In this study, we analyzed gene expression microarray profile of MCF7 breast cancer cells treated by cisplatin in two steps.
\end{abstract}

Materials and Methods: Data set encoded GSE28274 were used, which contains six sets of data regarded to control cells, cells treated in the step of glycolysis and onset of cell death impedance. The cell groups were compared to detect differentially expressed genes with a p-value less than 0.05 and a $\log 2$ Fold change more than \pm 1.2 by Using GEO2R tool of NCBI, The networks of the genes were constructed using STRING database and visualized by Gephi software. Hub genes were detected by network analysis. Functional annotation and biological pathways were determined by DAVID database.

Results: 626 genes are common in both steps. Up-regulated genes had larger percentage of the genes but most of the hubs were up-regulated. KEGG pathway and Gene Ontology biological process clearly showed the role of differentially expressed genes signaling process and apoptosis.

Conclusions: In conclusion, involved genes in signaling pathways are main ones in response to cisplatin and in both glycolysis and impedance steps there are some identical hub genes with similar activities and biological pathways. 